\title{
Association of pneumothorax and hypotension with intraventricular haemorrhage
}

\author{
Dariush Mehrabani, C W Gowen Jr, Arthur E Kopelman
}

\begin{abstract}
To test the hypothesis that acute hypotension resulting from pneumothorax would be associated with severe brain injury (grade 3 or 4 intraventricular haemorrhage), 67 very low birthweight (VLBW) infants of 32 weeks' gestation or less with respiratory distress syndrome and pneumothorax were studied. Thirty six had pneumothorax associated with systemic hypotension and 31 had pneumothorax with normal blood pressure. The groups were similar in gestational age and severity of their respiratory distress syndrome. Thirty two of $\mathbf{3 6}$ of infants with pneumothorax associated with hypotension (89\%) had grade 3 or 4 intraventricular haemorrhage. This percentage was significantly greater than the percentage for infants with pneumothorax and normal blood pressure (three of 31, 10\%). The risk ratio for grade 3 or 4 intraventricular haemorrhage for infants with pneumothorax associated with hypotension was 9.8 compared with neonates with pneumothorax and normal blood pressure. These observations are consistent with the hypothesis that systemic hypotension and cerebral hypoperfusion are important factors leading to intraventricular haemorrhage in VLBW infants.
\end{abstract}

Pulmonary air leak is a common complication in very low birthweight (VLBW) infants, occurring in up to $35 \%$ of those being ventilated for respiratory distress syndrome. ${ }^{12}$ Several reports have indicated that pneumothorax is often associated with, or followed by, intraventricular haemorrhage ${ }^{3-7}$ but other studies have not reported an increased risk of intraventricular haemorrhage. ${ }^{89}$ None of these studies, however, commented on the severity of symptoms caused by the pneumothorax or whether the pneumothorax was accompanied by systemic hypotension. A small pneumothorax may be asymptomatic or mildly symptomatic, but if the pneumothorax is associated with appreciably raised intrathoracic pressure then cardiac filling, cardiac output, and systemic blood pressure will fall. ${ }^{10-12}$ If this happens cerebral infarction is seen as the primary event, with intraventricular haemorrhage not occurring until after the systemic blood pressure has been raised to, or above, normal levels. ${ }^{11} 12$

In this study we tested the hypothesis that a pneumothorax with systemic hypotension would increase the likelihood of severe intraventricular haemorrhage in VLBW infants being ventilated for 'respiratory distress syndrome. Episodes of pneumothorax after which the blood pressure remained normal would be less likely to result in severe intraventricular haemorrhage.

We studied the incidence of grades 3 and 4 intraventricular haemorrhage among VLBW infants with pneumothorax that had caused hypotension, and compared it with the incidence of intraventricular haemorrhage in infants with pneumothorax and normal blood pressure.

\section{Patients and methods}

We retrospectively identified 82 infants from the records of our neonatal intensive care unit who were admitted between 1 January 1983 and 31 December 1986, were of 32 weeks' gestation or less, required mechanical ventilation for respiratory distress syndrome, and developed a pneumothorax. Fifteen were excluded from the study: five with hypoplastic lungs, one in whom the pneumothorax occurred as a terminal event, one in whom the pneumothorax was secondary to a thoracotomy for ligation of a patent ductus arteriosus, and two with congenital defects of the central nervous system. In addition, six others were excluded because they did not have a cranial ultrasound scan carried out after their pneumothorax, or because they had not undergone necropsy to see if there was an intraventricular haemorrhage.

The remaining 67 infants were studied. Two of the authors (DM, CWG) reviewed each neonate's chart to find out if the pneumothorax was associated with systemic hypotension. Blood pressure was monitored continuously by a transducer attached to an indwelling umbilical arterial catheter. Systemic hypotension, defined as a sudden fall in the mean blood pressure to less than two standard deviations below the mean for age $\left(30 \mathrm{~mm} \mathrm{Hg}\right.$ or less, ${ }^{13}$ ) resulted from a pneumothorax in 36 infants. An additional 31 infants had a pneumothorax but did not develop hypotension. Cranial ultrasound scans were independently scored according to the Papile system by a radiologist and a neonatologist (AEK) who did not know the medical history. ${ }^{3}$ The most severe grade of intraventricular haemorrhage on any ultrasound scan was used for the infant's score. For infants who died $(n=4)$ before ultrasound examination, the severity of intraventricular haemorrhage was determined at necropsy.

For each infant we recorded birth weight, sex, race, gestational age, Apgar score at one and five minutes, obstetric complications, time of first pneumothorax, and ventilator settings both at 12 hours of age and immediately before the first pneumothorax. The total number of 
days on the ventilator and being treated with oxygen, and the mortality, were also noted.

The incidence of intraventricular haemorrhage was determined for each group. Data were compared by the $\chi^{2}$ test and the unpaired Student's $t$ test. The relative risks for intraventricular haemorrhage for each group were calculated to answer the following question: is pneumothorax with systemic hypotension associated with an increased incidence of grades 3 and 4 intraventricular haemorrhage compared with pneumothorax during and after which the blood pressure remains normal?

\section{Results}

Although the infants in the two groups were of similar gestational age $(28.6(1.8)$ weeks compared with $29 \cdot 1(2 \cdot 2)$ weeks) the mean birth weight of the hypotensive infants (1026 (293) was lower than that of the group with normal blood pressure $(1250$ (356) $\mathrm{g}, \mathrm{p}<0.01)$ (table 1 ). In addition there were more boys in the hypotensive group ( 25 of $36,69 \%$ ) than in the group with normal blood pressure (15 of 31 , $42 \%)(p<0.03)$.

The average age of occurrence of the first pneumothorax was 29 hours (range 4-127) and 32 hours (range 2-100) in the hypotensive and normotensive groups, respectively. The pneumothorax was bilateral in about half the infants in each group; pneumothorax was right sided in about $66 \%$ of the unilateral pneumothoraces in each group.

Mean baseline measurements of blood pressure before the pneumothoraces were similar in the two groups (table 2). In all the infants who became hypotensive the mean arterial pressure fell when the pneumothorax occurred from $40(7 \cdot 1) \mathrm{mm} \mathrm{Hg}$ to $19 \cdot 2(10 \cdot 1)$ $\mathrm{mm} \mathrm{Hg}(\mathrm{p}<0.001)$. Mean blood pressures did not change significantly during the pneumothorax in the normotensive group.

After the pneumothorax the arterial oxygen tension fell significantly (from $9.73 \mathrm{kPa}$ to $7.07 \mathrm{kPa}, \mathrm{p}<0.05$ ) and the arterial carbon dioxide tension rose (from $4.80 \mathrm{kPa}$ to $6.67 \mathrm{kPa}$, $\mathrm{p}<0.05)$ in the hypotensive infants, but did not change significantly in the normotensive infants (from $8.66 \mathrm{kPa}$ to $8.13 \mathrm{kPa}$ and from $5.33 \mathrm{kPa}$ to $6.00 \mathrm{kPa}$, respectively).

There were no significant differences between the groups in ventilator mean airway pressure at 24 hours of age, but a significantly higher fractional inspiratory oxygen $\left(\mathrm{F}_{\mathrm{I}} \mathrm{O}_{2}\right)$ was required in the hypotensive infants $(0 \cdot 75$ compared with $0.62, p<0.05)$. There was no significant difference between the groups in mean airway pressure just before pneumothorax.

\section{RELATIONSHIP BETWEEN INTRAVENTRICULAR} HAEMORRHAGE AND PNEUMOTHORAX

The incidence of intraventricular haemorrhage for each study group is shown in the figure. Only one infant (3\%) in the hypotensive group had a normal cranial ultrasound scan compared with 24 infants (77\%) in the group with normal blood pressure. Thirty two infants, $89 \%$ of those in the hypotensive group, had grade 3 or 4 haemorrhages. The increased incidence of grade 3 or 4 intraventricular haemorrhage in this group of infants with pneumothorax and hypotension is highly significant compared with the group whose blood pressure remained normal ( 3 of $31,10 \%, p<0.01)$. The relative risk of grade 3 or 4 intraventricular haemorrhage was $\mathbf{9 \cdot 8}$ for

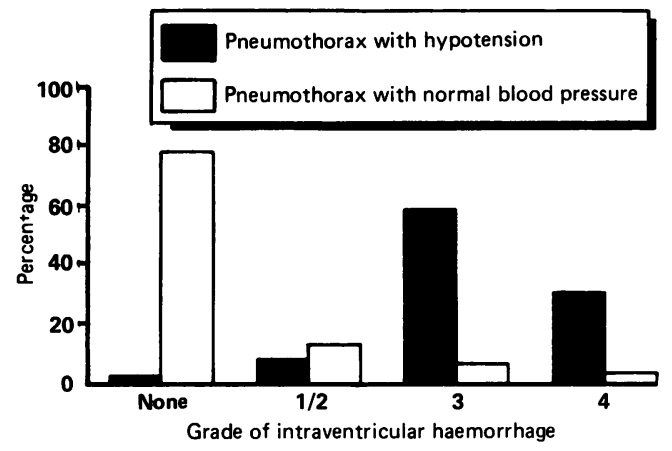

Infants with pneumothorax and hypotension had a higher incidence of grade 3 and 4 intraventricular haemorrhage. $\left({ }^{*} p<0 \cdot 01\right)$.

Table 1 Clinical characteristics of the study groups

\begin{tabular}{|c|c|c|c|}
\hline & $\begin{array}{l}\text { Pneumothorax with } \\
\text { hypotension }(n=36)\end{array}$ & $\begin{array}{l}\text { Pneumothorax with } \\
\text { normal blood } \\
\text { pressure }(n=31)\end{array}$ & $\overline{p \text { Value }}$ \\
\hline $\begin{array}{l}\text { Mean (SD) birth weight (g) } \\
\text { Mean (SD) gestational age (weeks) } \\
\text { Mean Apgar scores at } 1 \mathrm{~min} / 5 \mathrm{~min} \\
\text { No (\%) boys } \\
\text { No (\%) black infants } \\
\text { No (\%) born in hospital } \\
\text { No (\%) vaginal births } \\
\text { No (\%) with breech presentation } \\
\text { No (\%) with rupture of membranes for } \geqslant 24 \text { hours } \\
\text { No (\%) with respiratory distress syndrome grade } 3 \text { or } 4\end{array}$ & $\begin{array}{l}1026(293) \\
28 \cdot 6(1 \cdot 8) \\
3 / 5 \\
25(69) \\
15(42) \\
22(61) \\
24(67) \\
6(17) \\
6(17) \\
21(58)\end{array}$ & $\begin{array}{l}1250(356) \\
29 \cdot 1(2 \cdot 2) \\
3 / 5 \\
15(48) \\
18(58) \\
21(68) \\
12(39) \\
6(19) \\
5(16) \\
13(42)\end{array}$ & $\begin{array}{l}<0.01 \\
0.3 \\
0.07 \\
0.6 \\
0.6 \\
0.02 \\
0.8 \\
0.9 \\
0.2\end{array}$ \\
\hline
\end{tabular}

Table 2 Mean (SD) arterial blood pressure ( $\mathrm{mm} \mathrm{Hg}$ )

\begin{tabular}{llll}
\hline & Before pneumothorax & During pneumothorax & $\begin{array}{l}\text { After recovery } \\
\text { of pneumothorax }\end{array}$ \\
\hline $\begin{array}{l}\text { Pneumothorax with hypotension } \\
\begin{array}{l}\text { Pneumothorax with } \\
\text { normal blood pressure }\end{array}\end{array}$ & $40 \cdot 0(7 \cdot 1)^{*}$ & $19 \cdot 2(10 \cdot 1)^{*}$ & $37 \cdot 9(7 \cdot 8)$ \\
\hline p<0.001 & $36 \cdot 8(4 \cdot 8)$ & $36 \cdot 3(4 \cdot 7)$ & $37 \cdot 3(4 \cdot 3)$ \\
\hline
\end{tabular}


infants who became hypotensive compared with those whose blood pressure remained normal.

Because the study was retrospective we were not able to control for the timing of the cranial ultrasound scans. We therefore cannot say whether the pneumothorax preceded the intraventricular haemorrhage in all cases. Nevertheless, 17 infants in the hypotensive group had cranial ultrasound scans carried out both before, and within 24 hours after, their pneumothorax. Before the pneumothorax 10 of these 17 infants had normal cranial ultrasound scans and seven had either a grade 1 or grade 2 intraventricular haemorrhage. On repeat cranial ultrasound examination of the same infants after the pneumothorax, none had a normal cranial ultrasound, one had a grade 1-2 intraventricular haemorrhage, and 16 had grade 3 or 4 intraventricular haemorrhages. Though we cannot be certain that pneumothorax with a fall in blood pressure preceded the intraventricular haemorrhage in all cases, we believe that the observation in these 17 infants make it likely that severe intraventricular haemorrhage followed the development of pneumothorax.

\section{MORTALITY AND MORBIDITY}

Mortality was significantly higher in the hypotensive group ( 15 of $36,42 \%$ compared with 3 of $31,10 \% ; \mathrm{p}<0.005)$. Among the surviving infants, there was no difference between groups in the length of time the infants received assisted ventilation or supplemental oxygen. Residual brain damage was more common among the surviving hypotensive infants compared with the infants whose blood pressure remained normal; nine of 36 (25\%) compared with none of three of survivors developed unilateral periventricular leucomalacia with porenchephaly $(p<0.005)$, and 19 of $36(53 \%)$ compared with one of 31 (3\%) developed ventriculomegaly $(p<0.01)$.

\section{Discussion}

Intraventricular haemorrhage occurs in about $35 \%$ of premature neonates weighing less than 1500 at birth, and is a serious cause of mortality and morbidity. ${ }^{3}$ In addition, one recent report showed a significant association between hypotension and cerebral lesions or death. ${ }^{12}$ Intraventricular haemorrhage originates in the capillary network of the germinal layer, which is highly vascular and poorly supported. The pathogenesis of intraventricular haemorrhage is complex, but its occurrence is greatest in VLBW infants with respiratory distress syndrome. $^{14}$

A close association between pneumothorax and the occurrence and extension of intraventricular haemorrhage has been reported previously by Dykes et al who found that if a premature infant had an alveolar rupture, the risk of intraventricular haemorrhage was increased 2.5-fold compared with similar infants without alveolar rupture. ${ }^{5}$ Of all the risk factors studied, they found that the presence of pneumothorax had the strongest association with the later development of intraventricular haemorrhage. Lipscomb et $a l,{ }^{4}$ Hill et $a l,{ }^{6}$ and Thorburn et $\mathrm{al}^{15}$ also reported a close association between the development of a pneumothorax and a later intraventricular haemorrhage. Cooke, ${ }^{8}$ and Van De Bor et $a l,{ }^{9}$ however, did not find an increased incidence of intraventricular haemorrhage in premature infants with pneumothorax. The apparent discrepancy among these previous studies might be caused by differences in the severity of the pneumothoraces in the populations studied.

Pneumothorax in an infant can be virtually asymptomatic or may be associated with severe circulatory disturbances including hypotension and hypoperfusion. When severe pneumothorax leads to inadequate cardiac filling and systemic hypotension (air block syndrome) cerebral ischaemia may be induced, with the arterial watershed zones in the periventricular regions of the brain being particularly prone. ${ }^{11}$

Published data about the effect of pneumothorax on arterial blood pressure are somewhat conflicting. Hypotension secondary to pneumothorax has been clearly shown in both animal and human studies. ${ }^{212}$ 16-19 Other studies, however, have reported either no change in the arterial blood pressure or an increase in the arterial blood pressure with pneumothorax. ${ }^{6} 20$ It is likely that the effect of pneumothorax on arterial blood pressure in both animals and humans can be explained by the difference in the severity of the pneumothoraces studied. When there is sufficient intrathoracic pressure to compress the intrathoracic vessels severely, systemic hypotension will result (air block syndrome).

Our study has shown that $89 \%$ of our 36 infants with pneumothorax resulting in systemic hypotension developed grade 3 or 4 intraventricular haemorrhage. This percentage was significantly greater than for infants with pneumothorax that was not associated with hypotension $(9 \%)$. There was also a higher mortality among the infants with pneumothorax and hypotension than among infants with pneumothorax but no fall in blood pressure (42\% compared with $10 \%, p<0.005)$. In addition, infants with pneumothorax resulting in hypotension had a higher incidence of periventricular leucomalacia with porencephaly ( $25 \%$ compared with $0 \%, \mathrm{p}<0.01$ ) and ventricular dilation ( $53 \%$ compared with $3 \%, \mathrm{p}<0 \cdot 01$ ). The development of periventricular leucomalacia and ventricular dilatation in these infants is consistent with them having experienced an episode of severe cerebral ischaemia or infarction, or both.

One possible problem with this comparison is that the infants with pneumothorax associated with hypotension had a lower mean birth weight and there was a preponderance of boys. The groups were of similar gestational age, however, and the severity of their respiratory distress syndrome was similar (as shown by similar mean airway pressures on assisted ventilation and similar findings on chest radiographs. We are unable to say definitively that their higher incidence of grade 3 and 4 intraventricular haemorrhage is solely the result of their 
pneumothorax with associated hypotension. Nevertheless, those infants who developed a pneumothorax with hypotension had an extremely high incidence of intraventricular haemorrhage $(89 \%)$, identifying them as a group at extreme risk. No other single factor or combination of risk factors has been as strongly predictive of severe intraventricular haemorrhage. We therefore believe that these results strongly suggest (but cannot prove directly) that the pneumothorax associated with hypotension was the cause of the haemorrhage.

These observations support the theory that cerebral hypoperfusion or ischaemia precedes development of severe intraventricular haemorrhage. ${ }^{11}$ Recent reports indicate that premature infants who develop hypotension in the first days of life are likely to have cerebral parenchymal injury or severe intraventricular haemorrhage, while infants of similar gestational age who are not hypotensive do not develop these injuries. ${ }^{11} 12$ These infants lack cerebral autoregulation so that hypotension results directly in cerebral hypoperfusion. ${ }^{11} \mathrm{We}$ believe it is possible that the cerebral hypoperfusion that accompanies the systemic hypotension causes infarction of both the periventricular white matter and the germinal matrix. Later, when the blood pressure and cerebral blood flow are raised to normal values, the germinal matrix capillaries rupture and germinal matrix or intraventricular haemorrhages develop.

In summary, we found that $89 \%$ of 36 VLBW infants with respiratory distress syndrome who developed a pneumothorax with hypotension also developed a grade 3 or 4 intraventricular haemorrhage. These severe degrees of intraventricular haemorrhage have been associated with early mortality or neurological handicap in survivors. Based on our results, additional prospective studies should be carried out to determine the incidence and severity of brain injury in VLBW infants after episodes of moderate and severe hypotension.
1 Hill RT, Rhodes PG. Pneumothorax and pneumomediastinum in infants with idiopathic respiratory distress syndrome receiving continuous positive airway pressure. Pediatrics 1975;55:493-6.

2 Ogata EB, Gregory GA, Kitterman JA, et al. Pneumothorax in the respiratory distress syndrome: incidence and effect of vital signs, blood gases, and pH. Pediatrics 1976;58:177-83.

3 Papile LA, Burstein J, Burnstein R, et al. Incidence and evolution of subependymal and intraventricular hemorrhage: a study of infants with birth weights less than 1500 grams. f Pediatr 1978;92:529-34.

4 Lipscomb AP, Thornburn RJ, Reynolds EOR, et al. Pneumothorax and cerebral haemorrhage in preterm infants. Lancet $1981 ; \mathbf{i}: 414-6$.

5 Dykes FD, Lazzara A, Ahmann P, et al. Intraventricular hemorrhage: a prospective evaluation of etiopathogeneses. Pediatrics 1980;66:42-9.

6 Hill A, Perlman MB, Volpe JJ. Relationship of pneumothorax to occurrence of intraventricular hemorrhage in the preto occurrence of intraventricular hemorrha
mature newborn. Pediatrics 1982;69:144-9.

7 Kopelman A, Wimmer J, Engelke S, et al. Severe intracerebral hemorrhage in preterm infants impact on mortality and neurologic status. In: Jones CT, Nathanielsz PW, eds. The physiological development of the fetus and newborm. Part 7: Perinatal physiology and clinical care. Orlando: Academic Press, 1985:777-82.

8 Cooke RWI. Factors associated with periventricular haemorrhage in very low birth weight infants. Arch Dis Child 1981;56:425-31.

9 Van De Bor M, Van Bel F, Lineman R, Ruys JH. Perinatal factors and periventricular-intraventricular hemorrhage in preterm infants. Am $\mathcal{J}$ Dis Child 1986;140:1125-30.

10 Macklin MT, Macklin CC. Malignant interstitial emphysema of the lungs and mediastinum as an important occult complication in many respiratory diseases and other complication in many respiratory diseases and other light of laboratory experiment. Medicine 1984;23:281.

11 Lou HC. The 'lost autoregulation hypothesis' and brain lesions in the newborn. Brain Dev 1988;10:143-6.

12 Miall-Allen VM, de Vries LS, Dubowitz LMS, Whitelaw AGL. Blood pressure fluctuation and intraventricular AGL. Blood pressure fluctuation and intraventricular
hemorrhage in the preterm infant of less than 31 weeks' hemorrhage in the preterm infant of
gestation. Pediatrics 1989;83:657-61.

13 Shortland DB, Evans DH, Levene MI. Blood pressure measurements in very low birth weight infants over the first week of life. F Perinat Med 1988;16:93-7.

14 Volpe JJ. Neurology of the newborm. 2nd Ed. Philadelphia: WB Saunders, 1987.

15 Thornburn RJ, Lipscomb AP, Stewart AL, et al. Timing and antecedents of periventricular hemorrhage and cerebral atrophy in very preterm infants. Early Hum Dev 1982;7: 221-38.

16 Easley CS, Wartman FS, Cruze M, et al. Effect of tension pneumothorax on regional brain blood flow in the neonatal piglet. Medical Science Research 1989;17:711-3.

17 Batton DG, Hellman J, Nardis EE. Effect of pneumothoraxinduced systemic blood pressure alteration on the cerebral circulation in newborn dogs. Pediatrics 1984;74:350-3.

18 Monin P, Vert P. Pneumothorax. Clin Perinatol 1978;5:

19 Brazy JE, Blackmon LR. Hypotension and bradycardia associated with air block in the neonate. $\mathcal{f}$ Pediatr 1977;90:796.

20 Simmons DH, Hemingway A, Ricchiuti N. Acute circulatory effects of pneumothorax in dogs. $\mathcal{F}$ Appl Physiol 1958;12: 257-60. 